\title{
骨肉瘤 X 线与 CT 和 MRI 影像特点及诊断价值
}

\section{Features and Diagnostic Value of X-ray, CT and MRI Images of Osteosarcoma 万章玉}

\author{
Zhangyu Wan
}

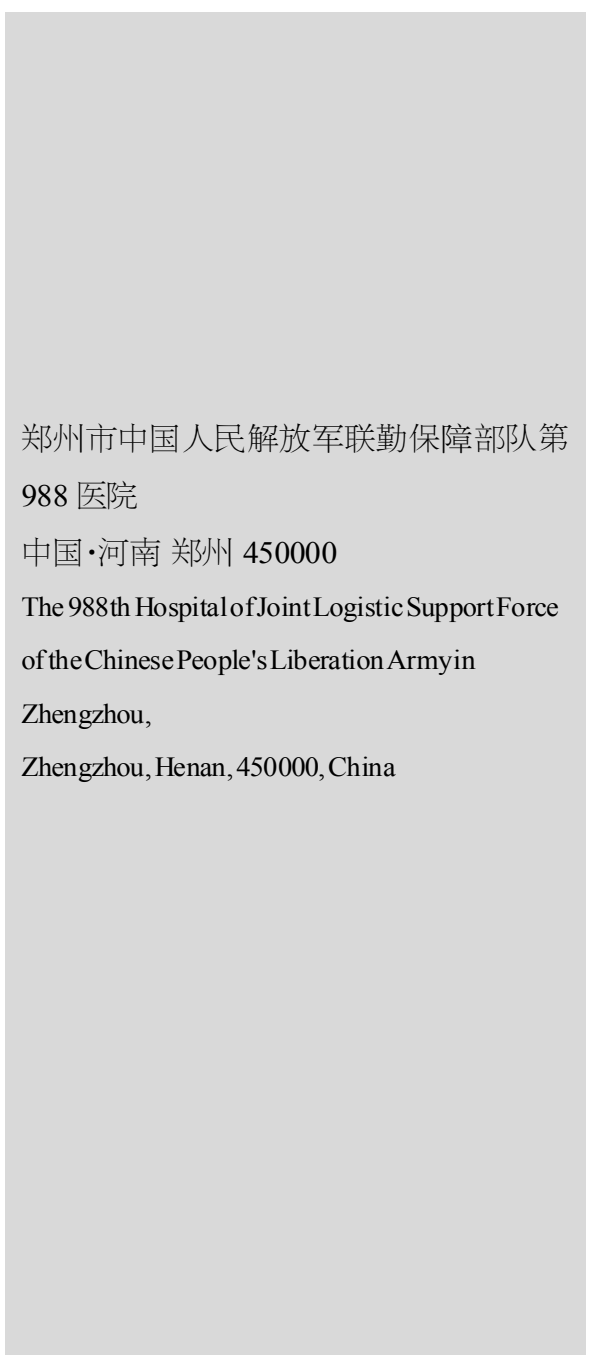

\section{1 引言}

骨肉瘤又名为骨生肉瘤, 主要包括原发性骨肉瘤和继发 性骨肉瘤两种类型。其中，原发性骨肉瘤的恶性肿瘤较为常 见,在青少年人群中发病率较高。而继发性骨肉瘤主要是从骨 肿瘤或其他骨科疾病演化而形成的。通常来说,骨肉瘤主要在 以膝关节为主的四肢长骨上较为多发。一旦肿瘤夢延至骨膜 下方, 并经骨面将骨膜剥离, 并在骨干和肿瘤的相接处生长出 三角形的反应性新生骨。骨肉瘤在发病过程中,病灶处会出现 程度不同的疼痛, 可摸出明显肿块, 严重时甚至伴有活动障 碍, 预后效果普遍较差。根据相关调查研究显示, 骨肉瘤疾病
应尽可能早期进行诊断。同时, 在诊断过程中, 应避免与软骨 肉瘤、纤维瘤发生混淆,防止出现误诊而影响患者的康复率。 本文采用回顾性分析, 以 X 线、CT 和 MRI 影像诊断判别骨肉 瘤的准确率为研究内容, 分析了其影响特点及诊断价值。

\section{2 资料和方法}

\section{1 一般资料}

本文选取我院于 2019 年 1 月一 2019 年 6 月收治的 30 例 骨肉瘤患者作为研究对象, 其中存在 16 位男患者, 14 位女患 者, 年龄为 $16 \sim 42$ 岁, 平均年龄为 $(26.5 \pm 3.1)$ 岁。存在 18 例患 者发病部位在股骨位置, 7 例患者发病部位在胫骨位置,4 例 
患者发病部位在忪骨位置, 1 例患者发病部位在尺骨位置。所 有患者在患病早期均伴有间歇性局部疼痛的症状, 并逐渐转 为持续性剧烈疼痛, 夜间尤甚。其中, 3 例患者皮肤红肿现象显 著,9 例患者病灶出现肿块,7 例患者活动受限。经由患者家属 同意, 对全体患者实施穿刺病理检查, 并经由手术确诊, 选择 性进行 X 线检查、CT 检查及 MRI 检查。

\section{2 检测方法}

$\mathrm{X}$ 线扫描:选用型号为 DWT 300A 的 X 线机, 并合理设置 扫描参数, 如电压应设为 $65 \mathrm{kV}$, 电流为 $100 \mathrm{mAs}$, 并将曝光时 间设为 $0.07 \mathrm{~s}$, 随后对患者局部病灶拍摄正位片和侧位片。

CT 扫描：选择型号为 GESigna16 的 CT 扫描仪, 并将层 厚、层距设为 $5 \mathrm{~mm}$, 设置电流为 $200 \mathrm{mAs}$, 对冠状位及矢状面进 行平扫。

MRI 扫描：扫描参数设置, 层厚设为 $3 \mathrm{~mm}$, 层距设为 $1.5 \mathrm{~mm}$, 进行冠状位、矢状位、轴位 $\mathrm{T} 1 \mathrm{~W} 1$ 和矢状位和冠状位 T2-STIR, 扫描层后轴位 $8 \mathrm{~mm}$, 冠状位和矢状位 $5 \mathrm{~mm}^{[1]}$ 。最后由 数名资深放射科医师采用双盲法阅片, 对 $\mathrm{X}$ 线、CT 和 MRI 影 像学资料进行分析。

\section{3 观察指标}

将病理学检查结果作为本次研究的黄金标准, 对 $\mathrm{X}$ 线、 CT 和 MRI 影像学资料的检查准确率进行分析, 并探讨采用上 述影像学检查方法检测骨肉瘤的影像学特点。

\section{4 统计学处理}

SPSS21.0 为本研究选用的统计学软件, 计量资料用 $\bar{x} \pm s$ 表示,计数资料用 $t$ 表示, $P<0.05$ 为差异有统计学意义。

\section{3 结果}

通过对 30 例骨肉瘤患者采用 X 线、CT 和 MRI 技术进行 影像学检查, 可得出 $\mathrm{X}$ 线检查的患者中确诊人数为 25 例, 准 确率为 $83.3 \%$; CT 检查的患者中确诊人数为 26 例, 准确率为 $86.7 \%$; MRI 检查的患者中确诊人数为 28 例, 准确率为 $93.3 \%$ 。 经过对比分析, 发现 3 种医疗影像诊断技术的准确率差异不具 备统计学意义 $(P>0.05)$ 。

在骨肉瘤征象的诊断中, 可知 MRI 在诊断骨质破坏、软组 织肿块方面的准确率分别为 $100 \%$ 和 $96.7 \%$, 相比 X 线和 CT 的诊断准确率要高; 而 CT 在诊断瘤骨方面的诊断率为 $98.7 \%$, 比 X 线和 MRI 的诊断准确率要高, 且差异均具备统计 学意义 $(P<0.05)$,具体数值如表 1 所示。

\section{4 讨论}

研究表明,骨肉瘤的发病率通常低于软骨肉瘤, 目前也未
表 $1 \mathrm{X}$ 线、CT 或 MRI 诊断骨肉瘤征象检查准确率

\begin{tabular}{c|c|c|c}
\hline 骨肉瘤征象 & X 线(例,\%) & CT(例, \%) & MRI(例,\%) \\
\hline 骨质破坏 & $15(50.0)$ & $24(80.0)$ & $30(100.0)^{\mathrm{ab}}$ \\
\hline 瘤骨 & $20(66.7)$ & $29(98.7)^{\mathrm{a}}$ & $17(56.7)^{\mathrm{b}}$ \\
\hline 骨膜反应 & $9(30.0)$ & $14(46.7)$ & $25(83.3)^{\mathrm{db}}$ \\
\hline 软组织肿块 & $6(20.0)$ & $14(46.7)$ & $29(96.7)^{\mathrm{db}}$ \\
\hline Codman 三角 & $11(36.7)$ & $19(63.3)$ & $13(43.3)$ \\
\hline
\end{tabular}

注: 与 $\mathrm{X}$ 线对比, ${ }^{\mathrm{a}} P<0.05$; 与 $\mathrm{CT}$ 对比, ${ }^{\mathrm{b}} P<0.05$ 。

能明确骨肉瘤的发病机制。部分学者认为骨肉瘤可能发生在 人体骨组织的任何部位，其中骨膜深层是最容易发生骨肉瘤 的部位。当肿瘤一旦产生,将会顺着骨膜向下不断蔓延,并引 起骨膜剥离, 生成新生骨, 容易发生严重恶化, 且经过临床治 疗后的预后效果较差。骨肉瘤没有明确的发病年龄限制, 在临 床上青少年为主要患病人群, 其中多数为中央型骨肉瘤。

为了诊断骨肉瘤, 需要医师结合患者的临床症状, 并根据 影像学检查结果进行诊断, 若想进一步确诊, 则需采用穿刺病 理检查或手术进行辅助。X 线、CT 和 MRI 影像诊断技术中, X 线具有较高的检查分辨率, 同时具有操作简便、价格低廉的优 势, 在临床诊断骨肉瘤方面具有较为广泛的应用范围。X 线检 查的劣势在于其密度分辨率较低, 容易导致影像中存在重叠现 象, 不利于确认患者病情。CT 检查则具有更高的密度分辨率, 能够清楚地显示骨肉瘤患者的骨质破坏情况、软组织肿块等 状态, 并能够在临床明确显示瘤骨的位置和钙化情况, 并帮助 医师了解肿瘤内部结构和周围组织受累情况。MRI 检查能够 实现多序列扫描,具有较高的组织分辨率, 但其在检查骨肉瘤 患者病灶影像时, 难以获取显著的特征, 因此其检查的目的主 要是显示肿瘤浸润及范围情况, 为临床提供可靠数据 ${ }^{[2]}$

\section{5 结语}

综上所述，在对本院 2019 年 1 月一 6 月 30 例骨髓瘤患 者进行影像学检验时, $\mathrm{X}$ 线、CT 和 MRI 检查的诊断准确率分 别为 $83.3 \% 、 86.7 \% 、 93.3 \%$ 。因此, $X$ 线影像检查方式在骨肉瘤 的临床检验方面的应用更为普遍, CT 在检查骨肉瘤患者骨质 破坏情况方面准确率较高, MRI 检查则能够明确显示出肿瘤 周围组织及肿瘤浸润情况，联合使用上述 3 种医学影像检查 方式,能够有效提升骨肉瘤临床诊断的准确性 ${ }^{[3]}$ 。

\section{参考文献}

[1]上官建伟,梁俊芳,肖新广, 等.骨肉瘤 X 线与 CT 和 MRI 影像特 点及诊断价值 $[\mathrm{J}]$. 医药论坛杂志, 2015,36(11):17-19.

[2]张汉荣.X 线、CT 及 MRI 对骨肉瘤的诊断对比分析 [J].临床医 药文献,2015,2(26):5517.

[3] 彭俊敏.比较分析 X 线、CT、MRI 诊断骨肉瘤的临床价值 [J].大 家健康(学术版),2015,9(16):57-58 